\title{
Spin-exchange and dipole relaxation rates in atomic hydrogen : rigorous and simplified calculations
}

\section{Citation for published version (APA):}

Stoof, H. T. C., Koelman, J. M. V. A., \& Verhaar, B. J. (1988). Spin-exchange and dipole relaxation rates in atomic hydrogen : rigorous and simplified calculations. Physical Review B: Condensed Matter, 38(7), 4688-4697. https://doi.org/10.1103/PhysRevB.38.4688

DOI:

10.1103/PhysRevB.38.4688

Document status and date:

Published: 01/01/1988

\section{Document Version:}

Publisher's PDF, also known as Version of Record (includes final page, issue and volume numbers)

\section{Please check the document version of this publication:}

- A submitted manuscript is the version of the article upon submission and before peer-review. There can be important differences between the submitted version and the official published version of record. People interested in the research are advised to contact the author for the final version of the publication, or visit the $\mathrm{DOI}$ to the publisher's website.

- The final author version and the galley proof are versions of the publication after peer review.

- The final published version features the final layout of the paper including the volume, issue and page numbers.

Link to publication

\section{General rights}

Copyright and moral rights for the publications made accessible in the public portal are retained by the authors and/or other copyright owners and it is a condition of accessing publications that users recognise and abide by the legal requirements associated with these rights.

- Users may download and print one copy of any publication from the public portal for the purpose of private study or research.

- You may not further distribute the material or use it for any profit-making activity or commercial gain

- You may freely distribute the URL identifying the publication in the public portal.

If the publication is distributed under the terms of Article 25fa of the Dutch Copyright Act, indicated by the "Taverne" license above, please follow below link for the End User Agreement:

www.tue.nl/taverne

Take down policy

If you believe that this document breaches copyright please contact us at:

openaccess@tue.nl

providing details and we will investigate your claim. 


\title{
Spin-exchange and dipole relaxation rates in atomic hydrogen: Rigorous and simplified calculations
}

\author{
H. T. C. Stoof, J. M. V. A. Koelman, and B. J. Verhaar \\ Department of Physics, Eindhoven University of Technology, NL-5600 MB Eindhoven, The Netherlands
}

(Received 2 July 1987)

\begin{abstract}
We calculate the magnetic field and temperature dependence of the rates for all two-body spinexchange and dipolar transitions among hyperfine levels in cryogenic $\mathbf{H}$ gas by means of the coupled-channels method. A description of this method and its practical application is presented. A simple interpretation of the rates is given, in some cases with associated simple closed-form formulas, based on the degenerate-internal-states approximation.
\end{abstract}

\section{INTRODUCTION}

In the past few years spin-polarized atomic hydrogen gas at subkelvin temperatures has attracted a great deal of attention. Already at lower densities the exceptional properties of this gas have shown up via the observation of spin waves. ${ }^{1}$ They have also been exploited in successful attempts to construct subkelvin $\mathrm{H}$ masers, ${ }^{2}$ with the aim of improving upon the frequency stability of existing atomic frequency standards. The primary interest, however, is still associated with the achievement of the temperature-density regime where effects due to Bose degeneracy are expected to show up. Although Bose- or Fermi-degenerate systems have already been studied extensively in various parts of physics, atomic hydrogen gas promises to be exceptional also from this point of view. In nuclear matter, for instance, the role of occupied levels in damping nucleon collisions within the Fermi sea and thus in explaining the validity of the nuclear shell model is qualitatively clear, but not easily calculated quantitatively owing to the high nucleon density, which makes it difficult to disentangle pure blocking effects from threeand more-particle collisions. A similar remark applies to superfluid helium considered as a Bose-degenerate system.

Degeneracy in polarized atomic hydrogen promises to be realizable in circumstances where average interatomic distances are comparable to the de Broglie wavelength but much larger than the range of interatomic forces, so that the macroscopic occupation of single-particle states will reveal itself in (relaxation and transport) properties describable with pure two-body collisions. The possibility, at least in principle, of independently controllable density and temperature is another attractive feature of Bose-degenerate atomic hydrogen, which distinguishes it from other systems like nuclear matter, superfluid helium, or the electron gas in metals.

Experimental and theoretical work on this topic has had a strong impetus recently by the idea of confining the polarized atomic gas in a magnetic trap, thus avoiding the decay of atomic density by three-body collisions at the helium-covered walls of a gas cell. In a static trap of this kind, ${ }^{3}$ however, other decay modes arise through two-body collisions of atoms in the two highest $1 \mathrm{~s}$ hyperfine states $|c\rangle$ and $|d\rangle$, leading to depolarization via the formation of atoms in the lowest states $|a\rangle$ and $|b\rangle$. A coupled-channels calculation of the associated rates has been presented by Lagendijk et al ${ }^{4}$

The purpose of the present paper is threefold. (1) To describe in a more complete form the coupled-channels method, which has been used for the calculation of the H-maser frequency shift ${ }^{5}$ and of the above-mentioned relaxation rates, both due to $\mathbf{H}+\mathbf{H}$ collisions. (2) To present new values for these rates based on a more accurate numerical calculation and a new $\mathrm{H}-\mathrm{H}$ singlet potential describing more precisely the experimental data on singlet binding energies. (3) To present a simplified description, in some cases with associated closed-form formulas, for the two-body rates in various regimes of temperature and magnetic field.

Apart from making clear how calculations described in previous papers on the $\mathrm{H}$-maser frequency shifts and on the rates in magnetic traps have been carried out, we believe that the new numerical values as well as the simple closed-form formulas will be extremely helpful for experimental groups working in these areas. For instance, the behavior of the $\mathbf{H} \uparrow$ gas in the first stages of confinement in a trap can be analyzed using the $T \neq 0$ rate constants. Also, for further work on the $\mathbf{H}$ maser, the present calculations are of interest. To devise strategies for circumventing the source of frequency instability of the cryogenic $\mathbf{H}$ maser, indicated in Ref. 5 , it is necessary to know the partial $|a\rangle,|b\rangle,|c\rangle$, and $|d\rangle$ densities of the hydrogen gas in the cavity. Information about rates for collisional transitions among the hyperfine levels is needed to determine these partial densities theoretically.

In Sec. II A we summarize some equations of scattering theory necessary to derive $S$ - and $T$-matrix elements for relaxation processes. In Sec. II B we consider the degenerate-internal-states approximation to these elements, and Sec. II C is devoted to the two-body rate equations. In Sec. III we describe our coupled-channels method in a more complete way than has hitherto been done. $^{6}$ The new results for the rates obtained in this way are presented in Sec. IV. We also consider the temperature dependence of some of them and in addition give a 
simplified description. Part of this has already been briefly pointed out in a previous paper on atomic deuterium in traps. ${ }^{7}$ Finally, some conclusions are given in Sec. V.

\section{TRANSITION AMPLITUDES AND RATES}

\section{A. $S$ matrix for coupled channels}

In this section we summarize some expressions, necessary in the following to calculate the rates and to find simplified expressions for some of them. Although the general structure of the final result for the $S$ matrix is obvious from the outset, its normalization has given rise to factor of 2 errors $^{6}$ in connection with $b b \rightarrow a b$ and $a b \rightarrow a a$ relaxation in the case of spin-polarized atomic hydrogen. For that reason it is necessary to define explicitly our conventions with respect to the $S$ matrix, as well as the precise normalization of the rate coefficients $G$ [cf. the factor $1+\delta_{\kappa \lambda}$ in Eq. (16)].

We start by considering the collision of two distinguishable particles with internal degrees of freedom, that are described by an internal Hamiltonian $H^{\text {int }}$ for each of the atoms with corresponding eigenstates:

$$
H^{\text {int }}|\alpha\rangle=\varepsilon_{\alpha}|\alpha\rangle \text {. }
$$

The total Hamiltonian of the two particles in the centerof-mass system is

$$
H=\frac{\mathrm{p}^{2}}{2 \mu}+\sum_{i=1}^{2} H_{i}^{\mathrm{int}}+V \equiv H_{0}+V .
$$

where $\mathbf{p}$ is the relative momentum, $\mu$ is the reduced mass, and $V$ represents the effective interaction among the particles, that may depend on the internal variables and is considered to be of finite range, i.e., $V(r)$ may be neglected beyond a certain value of $r$. Furthermore, we have introduced the free Hamiltonian $H_{0}$ with eigenstates $\left|\mathbf{p}_{\alpha \beta} \alpha \beta\right\rangle$ normalized as

$$
\left\langle\mathbf{p}_{\alpha^{\prime} \beta^{\prime}} \alpha^{\prime} \beta^{\prime} \mid \mathbf{p}_{\alpha \beta} \alpha \beta\right\rangle=\delta\left(\mathbf{p}_{\alpha^{\prime} \beta^{\prime}}-\mathbf{p}_{\alpha \beta}\right) \delta_{\alpha^{\prime} \alpha^{\prime}} \delta_{\beta^{\prime} \beta} .
$$

The associated eigenvalues are

$$
E_{\alpha \beta}=\frac{\mathbf{p}_{\alpha \beta}^{2}}{2 \mu}+\varepsilon_{\alpha}+\varepsilon_{\beta} .
$$

The scattering state $\left|\Psi_{\alpha \beta}^{(+)}\right\rangle$, that in a time-dependent picture develops out of the initial state $\left|\mathbf{p}_{\alpha \beta} \alpha \beta\right\rangle$, and incorporates all the available information of the scattering process has the familiar asymptotic behavior:

$$
\begin{array}{r}
\left\langle\mathbf{r} \mid \Psi_{\alpha \beta}^{(+)}\right\rangle \underset{r \rightarrow \infty}{\sim} \frac{1}{(2 \pi \hbar)^{3 / 2}}\left[e^{i \mathbf{p}_{\alpha \beta} \cdot \mathbf{r} / \hbar}|\alpha \beta\rangle\right. \\
+\sum_{\alpha^{\prime}, \beta^{\prime}} f_{\alpha^{\prime} \beta^{\prime}, \alpha \beta}\left(\mathbf{p}_{\alpha^{\prime} \beta^{\prime}}, \mathbf{p}_{\alpha \beta}\right) \\
\left.\times \frac{e^{i p_{\alpha^{\prime} \beta^{\prime}} r / \hbar}}{r}\left|a^{\prime} \beta^{\prime}\right\rangle\right],
\end{array}
$$

with $\mathbf{p}_{\alpha^{\prime} \beta^{\prime}} \equiv p_{\alpha^{\prime} \beta^{\prime}} \widehat{\mathbf{r}}$. For the derivation of this equation we refer to the literature. ${ }^{8,9}$ Furthermore, we have introduced the scattering amplitude for distinguishable particles

$$
\begin{aligned}
f_{\alpha^{\prime} \beta^{\prime}, \alpha \beta}\left(\mathbf{p}_{\alpha^{\prime} \beta^{\prime}}, \mathbf{p}_{\alpha \beta}\right) & \equiv-\frac{\mu(2 \pi \hbar)^{3}}{2 \pi \hbar^{2}}\left\langle\mathbf{p}_{\alpha^{\prime} \beta^{\prime}} \alpha^{\prime} \beta^{\prime}|V| \Psi_{\alpha \beta}^{(+)}\right\rangle \\
& \equiv-\frac{\mu(2 \pi \hbar)^{3}}{2 \pi \hbar^{2}}\left\langle\mathbf{p}_{\alpha^{\prime} \beta^{\prime}} \alpha^{\prime} \beta^{\prime}|T| \mathbf{p}_{\alpha \beta} \alpha \beta\right\rangle,
\end{aligned}
$$

and also defined the asymptotic magnitude of the momentum in channel $\left|\alpha^{\prime} \beta^{\prime}\right\rangle$ as

$$
p_{\alpha^{\prime} \beta^{\prime}}=\sqrt{2 \mu\left(E_{\alpha \beta}-\varepsilon_{\alpha^{\prime}}-\varepsilon_{\beta^{\prime}}\right)},
$$

the square root being positive real (open channels) or positive imaginary (closed channels).

Using the Lippmann-Schwinger equation ${ }^{8,9}$ for $\left|\Psi_{\alpha \beta}^{(+)}\right\rangle$ and the notation $G_{0}(z)=1 /\left(z-H_{0}\right)$ for the free resolvent operator, it is possible to derive the well-known ${ }^{8}$ equation for the $T$ operator in Eq. (5):

$$
T(z)=V+V G_{0}(z) T(z),
$$

that can, in principle, be solved for arbitrary complex $z$, representing the total energy available to the two-particle system.

Note that in an arbitrary plane-wave matrix element $\left\langle\mathrm{p}^{\prime} \alpha^{\prime} \beta^{\prime}|T(z)| \mathrm{p} \alpha \beta\right\rangle$ no specific relation among $\mathrm{p}^{\prime}, \mathrm{p}$, and $z$ needs to be fulfilled. This so-called off-shell $T$ matrix plays an important role in the three-body problem. ${ }^{8}$ In the present paper where only two-body collisions are considered, it will be more profitable to confine our attention to the on-shell $T$ (and $S$ ) matrix, but to consider it as a function of independent channel momenta $p_{\alpha^{\prime \prime} \beta^{\prime \prime}}$ of all coupled channels. We return to this in the second part of this section.

The expressions given so far were based on the $\mathbf{p}$ representation. For actual calculations it is far more convenient to work with angular momenta. We therefore transform to an angular momentum basis $|p l m\rangle$ in the momentum space of the particles, defined by

$$
\left\langle\mathbf{p}^{\prime} \mid p l m\right\rangle \equiv \frac{\delta\left(p-p^{\prime}\right)}{p p^{\prime}} Y_{l m}\left(\hat{\mathbf{p}}^{\prime}\right) .
$$

Equation (5) for the scattering amplitude can now be reformulated, introducing the $S$ matrix by

$$
\begin{aligned}
& S_{\alpha^{\prime} \beta^{\prime} l^{\prime} m^{\prime}, \alpha \beta l m}\left(p_{\alpha \beta}\right) \\
& \equiv \delta_{\alpha \alpha^{\prime}} \delta_{\beta \beta^{\prime}} \delta_{l l^{\prime}} \delta_{m m^{\prime}} \\
& \quad-2 \pi i \mu \sqrt{p_{\alpha \beta} p_{\alpha^{\prime} \beta^{\prime}}}\left\langle p_{\alpha^{\prime} \beta^{\prime}} l^{\prime} m^{\prime} \alpha^{\prime} \beta^{\prime}|T| p_{\alpha \beta} \operatorname{lm} \alpha \beta\right\rangle .
\end{aligned}
$$

The aforementioned $S$ matrix can be proven to be unitary, which corresponds to the conservation of particles. Furthermore, in the case that internal parity is unchanged $\left(\left|l-l^{\prime}\right|\right.$ is even), it turns out to be symmetrical. To define the $S$ matrix symmetrically in all cases, a phase-factor $i^{l}$ is to be added to every spherical harmonic. ${ }^{10}$ We thus find 


$$
\begin{aligned}
& \left\langle\mathbf{r} \mid \Psi_{\alpha \beta}^{(+)}\right\rangle \sim \frac{1}{(2 \pi \hbar)^{3 / 2}}\left[\sum_{\alpha^{\prime}, \beta^{\prime}} \sum_{l^{\prime}, m^{\prime}} \sum_{l, m} \frac{2 \pi i^{l^{\prime}}}{i \sqrt{p_{\alpha \beta} / \hbar}} \mid S_{\alpha^{\prime} \beta^{\prime} l^{\prime} m^{\prime}, \alpha \beta l m}\left(p_{\alpha \beta}\right) \frac{e^{i\left(p_{\alpha^{\prime} \beta^{r}} r / \hbar-l^{\prime} \pi / 2\right)}}{r \sqrt{p_{\alpha^{\prime} \beta^{\prime}} / \hbar}}\right. \\
& \left.+\delta_{\alpha \alpha^{\prime}} \delta_{\beta \beta^{\prime}} \delta_{l l^{\prime}} \delta_{m m^{\prime}} \frac{e^{-i\left(p_{\alpha^{\prime} \beta^{\prime}} r / \hbar-l^{\prime} \pi / 2\right)}}{r \sqrt{p_{\alpha^{\prime} \beta^{\prime}} / \hbar}}\right) \\
& \left.\times Y_{l m}^{*}\left(\widehat{\mathbf{p}}_{\alpha \beta}\right) Y_{l^{\prime} m^{\prime}}\left(\hat{\mathbf{p}}_{\alpha^{\prime} \beta^{\prime}}\right)\left|\alpha^{\prime} \beta^{\prime}\right\rangle\right] \text { as } r \rightarrow \infty \text {. }
\end{aligned}
$$

We are left with the generalization to identical particles. Because of our interest in atomic hydrogen we will confine ourselves to bosons. It turns out that Eq. (10) is again valid for the symmetrized wave function $\left.\left\langle\mathbf{r}|\mathcal{S}| \Psi_{\alpha \dot{\beta}}^{(}\right)\right\rangle, \quad \mathcal{S}=\left(1+\mathcal{P}_{12}\right)$, if we add a factor $2 / \sqrt{2-\delta_{\alpha \beta}}$ to the right-hand side and replace all channel states by symmetrized and normalized states $|p \operatorname{lm}\{\alpha \beta\}\rangle$ defined by

$$
|p \operatorname{lm}\{\alpha \beta\}\rangle=\frac{|p \operatorname{lm} \alpha \beta\rangle+(-1)^{l}|p \operatorname{lm} \beta \alpha\rangle}{\sqrt{2\left(1+\delta_{\alpha \beta}\right)}} .
$$

In addition, the right-hand side now contains symmetrized $S$-matrix elements $S_{\left\{\alpha^{\prime} \beta^{\prime}\right\} l^{\prime} m^{\prime},\{\alpha \beta\} l m}$ and corresponding Kronecker symbols $\delta_{\left\{\alpha^{\prime} \beta^{\prime}\right\},\{\alpha \beta\}}$. Equation (10) modified in this way is used in the discussion of the coupled-channels approach in Sec. III.

\section{B. Degenerate-internal-state approximation}

In a previous paper we already pointed out briefly the importance of neglecting in a specific way the differences between internal energy levels. ${ }^{7}$ Here we will formulate this approach in a more general form, applicable also for $T \neq 0$. Let us first consider exchange transitions. The general idea is to consider an angular momentum basis $T$-matrix element as a function of all channel momenta involved. In simplified notation

$$
T_{l_{f} l_{l}}\left(p_{1}, p_{2}, \ldots, p_{i}, \ldots, p_{f}, \ldots, p_{n}\right)
$$

and to split off factors $p_{i}^{l_{i}}$ and $p_{f}^{l_{f}}$ pertaining to the lowenergy dependence in the initial and final channels, respectively. The remaining factor is approximated by its (in general nonvanishing) value for $p_{1}=p_{2}=\cdots=p_{n}=p$, for which the limit $p \rightarrow 0$ may be taken, but also a common value $p \neq 0$ :

$$
\begin{aligned}
& T_{l_{f} l_{i}}\left(p_{1}, p_{2}, \ldots, p_{i}, \ldots, p_{f}, \ldots, p_{n}\right) \\
& \quad=\left(p_{f} / p\right)^{l_{f}}\left(p_{i} / p\right)^{l_{i}} T_{l_{f} l_{i}}(p, p, \ldots, p, \ldots, p, \ldots, p) .
\end{aligned}
$$

Note that for exchange transitions $l_{f}=l_{i}$. Note also that the degenerate-internal-states approximation (usually referred to as adiabatic but also as sudden approximation) is often thought to be a typical high-energy approximation, in the sense that all differences between internal energies should be small compared to the asymptotic kinetic energy. In such a case a choice for $p$ so that it is of the order of the asymptotic channel momenta, is preferable. We already pointed out in Ref. 7 , however, that this high-energy condition is too strong.

For dipolar transitions the degenerate-internal-states approximation does not work in the form (12). Due to the long range of the dipole interaction the low-energy behavior of dipolar $T$-matrix elements does not correspond to the above-mentioned powers of $p_{f}$ and $p_{i}$. It is possible, however, to implement the degenerate-internalstates approximation for dipolar transitions in a different way. In all cases a calculation to first order in $V^{d}$ is sufficiently accurate, i.e.,

$$
T_{\left\{\alpha^{\prime} \beta^{\prime}\right\} l^{\prime} m^{\prime},\{\alpha \beta\} l m}={ }_{c}\left\langle\psi_{\left\{\alpha^{\prime} \beta^{\prime}\right\} l^{\prime} m^{\prime}}^{(-)}\left|V^{d}\right| \psi_{\{\alpha \beta\} l m}^{(+)}\right\rangle_{c},
$$

the subscript $c$ indicating that the corresponding scattering state is distorted by central interactions only. The state $\left|\psi_{\left\{\alpha^{\prime} \beta^{\prime}\right\} l^{\prime} m^{\prime}}^{(-)}\right\rangle_{c}$ describes a time-reversed scattering process and therefore obeys incoming asymptotic boundary conditions. We now apply the degenerate-internalstates approximation to initial and final states separately, so that both can be expressed as linear combinations of pure singlet and triplet scattering states. We come back to this in Sec. IV B.

\section{Rate equations}

In this subsection we present expressions for two-body rate constants in terms of the symmetrized $S$-matrix elements introduced above. A safe way to derive these, from the point of view of avoiding factor of 2 identicalparticle errors, is to make use of a quantum Boltzmann equation that can be derived from the BBGKY hierarchy: ${ }^{5}$

$$
\begin{array}{r}
\frac{d}{d t} \rho_{\kappa^{\prime} \kappa}+i\left(\frac{\varepsilon_{\kappa^{\prime}}-\varepsilon_{\kappa}}{\hbar}\right) \rho_{\kappa^{\prime} \kappa}=n \sum_{\lambda} \sum_{\mu^{\prime}, v^{\prime}} \sum_{\mu, v} \rho_{\mu^{\prime} \mu} \rho_{v^{\prime} v}\left[\left(1+\delta_{\kappa^{\prime} \lambda}\right)\left(1+\delta_{\mu^{\prime} v^{\prime}}\right)\left(1+\delta_{\kappa \lambda}\right)\left(1+\delta_{\mu \nu}\right)\right]^{1 / 2} \\
\times\left\langle\frac { \pi \hbar ^ { 2 } } { 2 \mu q } \sum _ { l ^ { \prime } , m ^ { \prime } } \sum _ { l , m } \left( S_{\left\{\kappa^{\prime} \lambda\right\} l^{\prime} m^{\prime},\left\{\mu^{\prime} v^{\prime}\right\} l m}(q) S_{\{\kappa \lambda\} l^{\prime} m^{\prime},\{\mu v\} l m}(q)\right.\right. \\
\left.\left.-\delta_{\left\{\kappa^{\prime} \lambda\right\},\left\{\mu^{\prime} v^{\prime}\right\}} \delta_{l^{\prime} l} \delta_{m^{\prime} m} \delta_{\{\kappa \lambda\},\{\mu v\}}\right)\right\rangle_{\mathrm{th}} .
\end{array}
$$


Here $\rho$ represents the one-particle density matrix, normalized according to $\operatorname{Tr}(\rho)=1, n$ is the particle density, and $q$ the relative momentum of the colliding pair. The right-hand side contains a thermal average with respect to $q$. In a previous paper ${ }^{5}$ we used Eq. (14) with the coherence of the $a$ and $c$ states included $\left(\rho_{a c}=\rho_{c a}^{*} \neq 0\right)$ as a starting point to derive the spin-exchange frequency shift of the cryogenic $\mathbf{H}$ maser. The same equation may serve as a starting point to derive the two-body rate equations. In this case the hyperfine states contribute incoherently:

$$
n \rho_{\kappa^{\prime} \kappa}=n_{\kappa} \delta_{\kappa^{\prime} \kappa} .
$$

We use the unitarity of the $S$ matrix to express the elastic $S$-matrix element occurring in Eq. (14) in terms of inelastic ones. This leads to the well-known combination of "in" and "out" terms:

$$
\frac{d}{d t} n_{\kappa}=\sum_{\lambda} \sum_{\{\mu \nu\}}\left(1+\delta_{\kappa \lambda}\right)\left(G_{\mu \nu \rightarrow \kappa \lambda} n_{\mu} n_{v}-G_{\kappa \lambda \rightarrow \mu v} n_{\kappa} n_{\lambda}\right)
$$

in which the relaxation constants are given by

$$
\begin{aligned}
G_{\alpha \beta \rightarrow \alpha^{\prime} \beta^{\prime}}= & \left\langle\frac{\pi \hbar^{2}}{\mu q} \sum_{l^{\prime}, m^{\prime}} \sum_{l, m}\right| S_{\left\{\alpha^{\prime} \beta^{\prime}\right\} l^{\prime} m^{\prime},\{\alpha \beta\} l m}(q) \\
& \left.-\left.\delta_{\left\{\alpha^{\prime} \beta^{\prime}\right\} l^{\prime} m^{\prime},\{\alpha \beta\} l m}\right|^{2}\right\rangle_{\text {th }} .
\end{aligned}
$$

Equation (17) gives the desired relation between the macroscopic quantities $G_{\alpha \beta \rightarrow \alpha^{\prime} \beta^{\prime}}$ and the microscopic $S$ matrix, that can be used to calculate the rate constants of all possible exothermic transitions among the hyperfine states of atomic hydrogen. It is sufficient to consider only these transitions, because the rate constants of a process and its inverse are related by

$$
G_{\alpha \beta \rightarrow \alpha^{\prime} \beta^{\prime}}=G_{\alpha^{\prime} \beta^{\prime} \rightarrow \alpha \beta} e^{-\left(\varepsilon_{\alpha^{\prime}}+\varepsilon_{\beta^{\prime}}-\varepsilon_{\alpha}-\varepsilon_{\beta}\right) / k T} .
$$

\section{COUPLED-CHANNELS APPROACH}

We start by describing in more detail the effective Hamiltonian (2). Considered as a four-particle problem (two electrons and two protons), a collision between hydrogen atoms is a complicated process. It is well known, ${ }^{6}$ however, that the problem can be considerably simplified by means of the Born-Oppenheimer and Shizgal approximations, which replace the total Hamiltonian by an effective expression (2) in which $H^{\text {int }}$ consists of the hyperfine and Zeeman interactions for each of the atoms:

$$
H_{i}^{\mathrm{int}}=\frac{a}{4} \boldsymbol{\sigma}_{i}^{e} \cdot \boldsymbol{\sigma}_{i}^{p}+\mu_{e} \mathbf{B} \cdot \boldsymbol{\sigma}_{i}^{e}-\mu_{p} \mathbf{B} \cdot \boldsymbol{\sigma}_{i}^{p} \quad(i=1,2)
$$

with $a$ the hyperfine constant, $\mu_{e}$ and $\mu_{p}$ the electron and proton magnetic moments, respectively, and $\sigma_{i}$ the Pauli spin matrices. The eigenstates of this internal Hamiltonian are conventionally denoted by $|a\rangle,|b\rangle,|c\rangle$, and $|d\rangle$ in order of increasing energy. In terms of electron $(\uparrow)$ and proton spins $(\uparrow)$ they have the form

$$
\begin{aligned}
& |a\rangle=\cos \theta|\downarrow \uparrow\rangle-\sin \theta|\uparrow \downarrow\rangle, \\
& |b\rangle=|\downarrow \downarrow\rangle, \\
& |c\rangle=\cos \theta|\uparrow \downarrow\rangle+\sin \theta|\downarrow \uparrow\rangle, \\
& |d\rangle=|\uparrow \uparrow\rangle,
\end{aligned}
$$

where $\theta$ follows from $\tan (2 \theta)=a /\left[2 B\left(\mu_{e}+\mu_{p}\right)\right]$.

Furthermore, $H$ contains the effective interaction

$$
V=V^{c}+V^{d} \text {. }
$$

We use the notation $V^{c}$ for the central (singlet and triplet) interactions

$$
V^{c}=V_{0}(r) \mathcal{P}^{(0)}+V_{1}(r) \mathcal{P}^{(1)}
$$

$\mathcal{P}^{(0)}\left(\mathcal{P}^{(1)}\right)$ denoting a projection operator on the subspace of singlet (triplet) states. The operator $V^{d}$ stands for the sum of the electron-electron and electron-proton magnetic dipole interactions. It is a scalar product of operators of rank 2:

$$
\begin{aligned}
V^{d}(\mathbf{r})= & -\frac{\mu_{0} \mu_{e}^{2}}{4 \pi r^{3}} \sum_{\mu=-2}\left[\frac{4 \pi}{5}\right)^{1 / 2}(-1)^{\mu} Y_{2,-\mu}(\hat{\mathbf{r}}) \Sigma_{2 \mu}^{e e} \\
& +2 \frac{\mu_{0} \mu_{e} \mu_{p}}{4 \pi r^{3}} \sum_{\mu=-2}^{2}\left(\frac{4 \pi}{5}\right)^{1 / 2}(-1)^{\mu} Y_{2,-\mu}(\hat{\mathbf{r}}) \Sigma_{2 \mu}^{e p} .
\end{aligned}
$$

The tensor operator $\Sigma^{i j}$ can be thought of as arising from the coupling of $\sigma^{i}$ and $\sigma^{j}$ to a spin operator of rank 2. As usual we leave out the proton-proton dipole term.

After this description of the effective Hamiltonian we turn to the problem of solving the time-independent Schrödinger equation. We introduce a fully symmetrical basis of so-called channel states $\{|\operatorname{rlm}\{\alpha \beta\}\rangle\}$, similar to Eqs. (8) and (11), and decompose $\left|\Psi^{(+)}\right\rangle$as

$$
\left|\Psi^{(+)}\right\rangle=\sum_{\{\alpha \beta\}} \sum_{l, m} \int_{0}^{\infty} d r r^{2} \frac{F_{l m\{\alpha \beta\}}(r)}{r} i^{l}|r \operatorname{lm}\{\alpha \beta\}\rangle .
$$

In coordinate space we thus find

$$
\left\langle\mathbf{r} \mid \Psi^{(+)}\right\rangle=\sum_{\{\alpha \beta\}} \sum_{l, m} \frac{F_{l m \mid \alpha \beta\}}(r)}{r} i^{l} Y_{l m}(\hat{\mathbf{r}})|\{\alpha \beta\}\rangle .
$$

Substituting this expression into the Schrödinger equation and projecting onto the state $|r \operatorname{lm}\{\alpha \beta\}\rangle$ gives the desired coupled-channels equation:

$$
\begin{aligned}
\left(-\frac{\hbar^{2}}{2 \mu} \frac{d^{2}}{d r^{2}}\right. & \left.+\frac{l(l+1) \hbar^{2}}{2 \mu r^{2}}+\varepsilon_{\alpha}+\varepsilon_{\beta}-E\right) F_{l m\{\alpha \beta\}}(r) \\
& =-\sum_{\left\{\alpha^{\prime} \beta^{\prime}\right\}} \sum_{l^{\prime}, m^{\prime}} C_{l m\{\alpha \beta\}, l^{\prime} m^{\prime}\left\{\alpha^{\prime} \beta^{\prime}\right\}}(r) F_{l^{\prime} m^{\prime}\left\{\alpha^{\prime} \beta^{\prime}\right\}}(r),
\end{aligned}
$$

in which the coupling matrix is defined by

$$
\begin{aligned}
& C_{l m\{\alpha \beta\}, l^{\prime} m^{\prime}\left\{\alpha^{\prime} \beta^{\prime}\right\}}(r) \frac{\delta\left(r-r^{\prime}\right)}{r r^{\prime}} \\
& \quad=i^{l^{\prime}-l}\left\langle r \operatorname{rm}\{\alpha \beta\}\left|\left(V^{c}+V^{d}\right)\right| r^{\prime} l^{\prime} m^{\prime}\left\{\alpha^{\prime} \beta^{\prime}\right\}\right\rangle .
\end{aligned}
$$


For spin states $\{\alpha \beta\}$ and $\left\{\alpha^{\prime} \beta^{\prime}\right\}$ with equal total $M_{F}$ the $V^{c}$ contribution, if it does not vanish, dominates by far that due to $V^{d}$. It is customary to indicate such processes as exchange transitions. Processes to which only $V^{d}$ contributes are indicated as dipolar transitions. This is the case, in particular, for $\Delta M_{F} \neq 0$ which requires a transfer of angular momentum among orbital and spin degrees of freedom. For even $l$ also the $\Delta M_{F}=0$ transitions with $M_{F} \neq 0$ belong to this category.

To discuss the numerical method for solving Eq. (26) we introduce a matrix notation

$$
\frac{d^{2}}{d r^{2}} \underline{F}(r)=\frac{2 \mu}{\hbar^{2}} \underline{C}(r) \underline{F}(r),
$$

where the columns of $\underline{F}$ represent a complete set of linearly independent solutions. The rows correspond to the mutually coupled channels $|\operatorname{lm}\{\alpha \beta\}\rangle$. Because of rotational symmetry around the magnetic field direction all of these have the same total magnetic quantum number $M=m+M_{F}$.

Equation (28) is numerically integrated using a

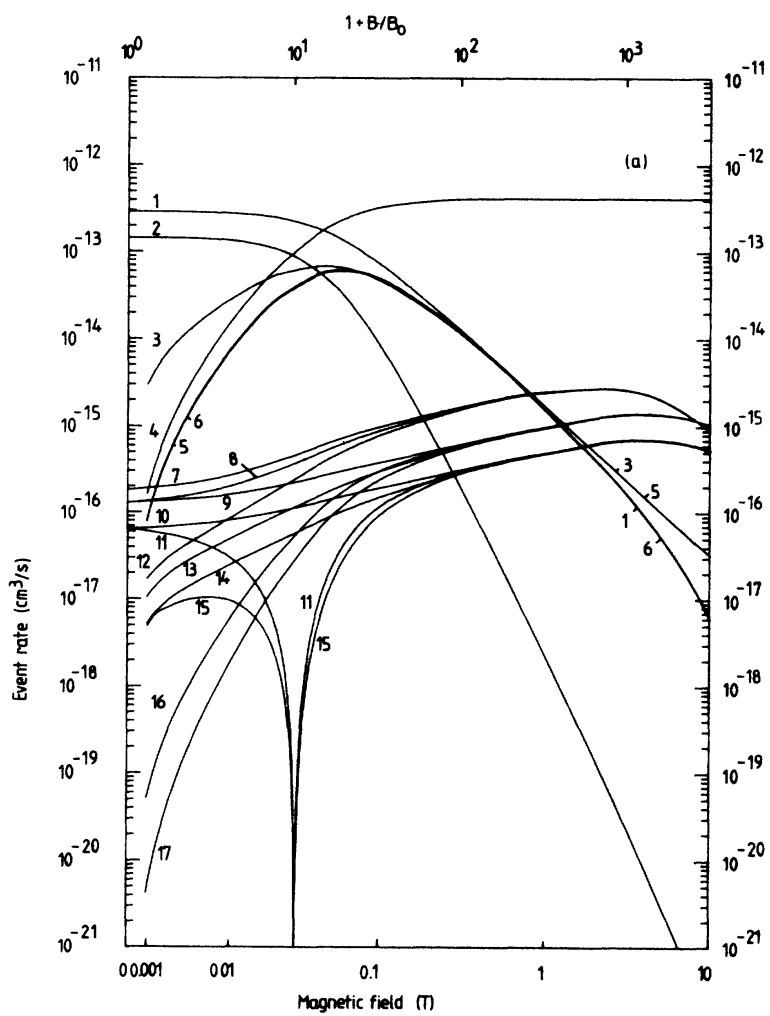

modified Numerov method. ${ }^{11}$ During the integration there is a danger of losing sufficient linear independence of the different solutions relative to the numerical noise level. This is caused by the "locally closed" channels, for which $C_{l m\{\alpha \beta\}, \operatorname{lm}\{\alpha \beta\}}(r)>0$, leading to exponential growth of one or more common row elements in all columns of $\underline{F}$ and therefore to numerically linearly dependent solutions. We solve this problem by transforming to new linear combinations of solutions at one or more values of $r$, thus restoring sufficient linear independence. ${ }^{12}$

From $F(r)$ we extract the $S$ matrix. To that end we introduce two diagonal matrices containing Hankel-like basis solutions of Eq. (28) with the right-hand side equal to 0:

$$
\begin{aligned}
I_{m n}(r) & =-\delta_{m n} \sqrt{p_{m} / \hbar i r h_{l_{m}}^{(-)}\left(p_{m} r / \hbar\right)} \\
& \sim-\delta_{m n} i \frac{e^{-i\left(p_{m} r / \hbar-l_{m} \pi / 2\right)}}{\sqrt{p_{m} / \hbar}} \text { as } r \rightarrow \infty, \\
O_{m n}(r) & =I_{m n}^{*}(r) .
\end{aligned}
$$

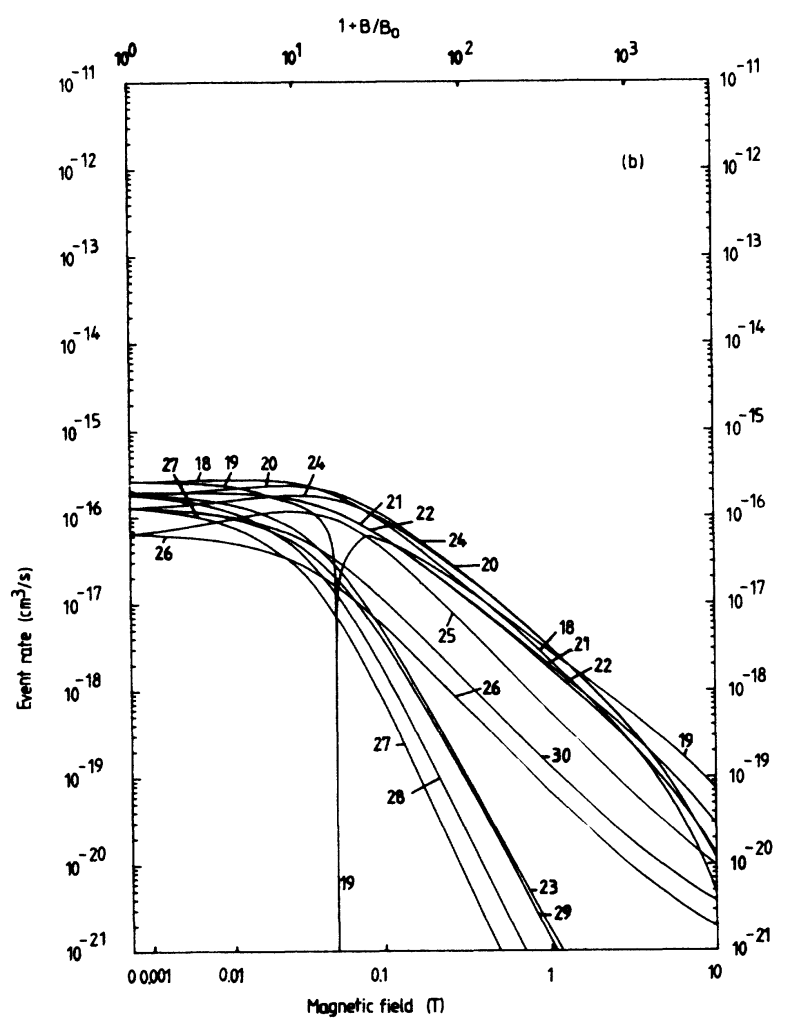

FIG. 1. $T=0$ spin exchange and dipolar relaxation event rates as a function of magnetic field. The horizontal scale shows a gradual transition from a linear to a logarithmic field dependence as we plot $1+B / B_{0}$ logarithmically. Here $B_{0}=a / 16 \mu_{B}=3.17 \times 10^{-3} T$ ( $\mu_{B}$ is the Bohr magneton), the factor $\frac{1}{16}$ ensuring a favorable separation in linear and logarithmic parts. The lower horizontal scale shows corresponding values for $B$. (a) The curves correspond to the following rates: (1): $b d \rightarrow a a,(2): c c \rightarrow a a,(3): c c \rightarrow b d,(4)$ : $b d \rightarrow a c,(5): c c \rightarrow a c,(6): a c \rightarrow a a,(7): d d \rightarrow a a,(8): c d \rightarrow a b,(9): d d \rightarrow a d,(10): b d \rightarrow a b,(11): c d \rightarrow a c,(12): c c \rightarrow b b,(13): b c \rightarrow b b$, (14): $c d \rightarrow b d$, (15): $a c \rightarrow a b,(16): a d \rightarrow a a,(17): c c \rightarrow b c$. (b) The curves correspond to the following rates: (18): $d d \rightarrow a c$, (19): $a c \rightarrow b b,(20): c d \rightarrow a a,(21): \quad b c \rightarrow a b,(22): c d \rightarrow a d,(23): b c \rightarrow a a,(24): c c \rightarrow a b,(25): b c \rightarrow a c,(26): b d \rightarrow a d,(27): b c \rightarrow a d,(28):$ $b b \rightarrow a a,(29): c c \rightarrow a d,(30): b b \rightarrow a b$. (c) The remaining relaxation rates, not shown in (a) and (b). Curves correspond to the following rates: (31): $d d \rightarrow c d,(32): a d \rightarrow a c$, (33): $b d \rightarrow b c$, (34): $a b \rightarrow a a,(35): c d \rightarrow c c,(36): b d \rightarrow b b,(37): a d \rightarrow a b,(38): c d \rightarrow b c,(39):$ $d d \rightarrow c c$. 


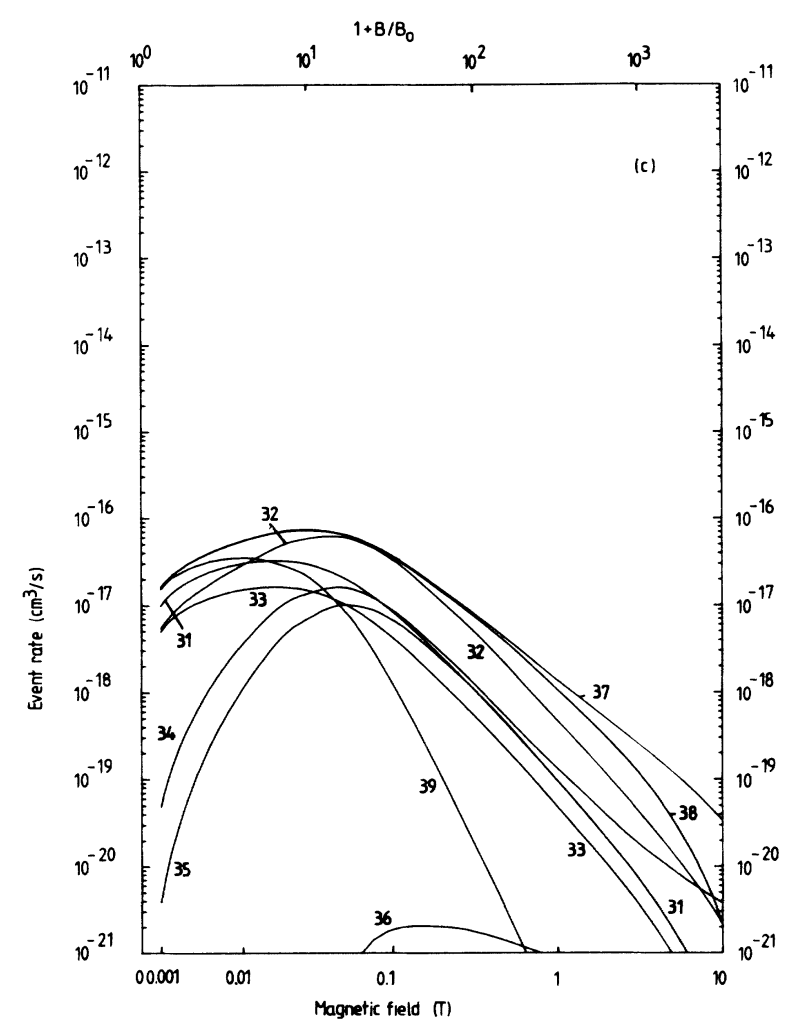

FIG. 1. (Continued).

Here $h_{l}^{(-)}(\rho)$ is a spherical Hankel function. Again $p_{m}$ is positive real or positive imaginary. With the help of these matrices the solution $\underline{F}(r)$ can be written as

$$
\underline{F}(r)=\underline{I}(r) \underline{A}(r)+\underline{Q}(r) \underline{B}(r) .
$$

Applying the method of variation of parameters ${ }^{13}$ results in a coupled set of first-order differential equations for the coefficient matrices:

$$
\begin{aligned}
& \frac{d}{d r} \underline{A}(r)=\frac{\mu i}{\hbar^{2}} \underline{O}(r) \underline{C}(r)[\underline{I}(r) \underline{A}(r)+\underline{O}(r) \underline{B}(r)], \\
& \frac{d}{d r} \underline{B}(r)=-\frac{\mu i}{\hbar^{2}} \underline{I}(r) \underline{C}(r)[\underline{I}(r) \underline{A}(r)+\underline{O}(r) \underline{B}(r)] .
\end{aligned}
$$

To find the $S$ matrix we integrate the coupled-channels equations (28) until the coefficient matrices in Eq. (30) have become $r$ independent. We then have

$$
\underline{F}(r)=\underline{I}(r) \underline{A}+\underline{Q}(r) \underline{B}=[\underline{I}(r)+\underline{Q}(r) \underline{S}] \underline{A} .
$$

This defines the $S$ matrix as $\underline{S} \equiv \underline{B A}^{-1}$. Comparison with the asymptotic behavior described below Eq. (10) leads to the conclusion that the open-channel submatrix of $\underline{S}$ so derived, is identical to the $S$ matrix to be calculated.
The application of the above-mentioned procedure would involve an integration to very large radii, because the dipole interaction decreases only as $r^{-3}$. Therefore, we restrict the integration of Eq. (28) to within a radius of $r_{1}$, beyond which the central interactions can be neglected and treat the dipole interaction in the region $r>r_{1}$ as a perturbation. We achieve this by defining a local $S$ matrix through the relation $\underline{S}(r)=\underline{B}(r) \underline{A}^{-1}(r)$ and deriving from Eq. (31) an integral equation for $\underline{S}(r)$ :

$$
\begin{aligned}
\underline{S}(r)=\underline{S}\left(r_{1}\right)-\frac{\mu i}{\hbar^{2}} \int_{r_{1}}^{r} d r^{\prime}\left[\underline{I}\left(r^{\prime}\right)+\underline{S}\left(r^{\prime}\right) \underline{Q}\left(r^{\prime}\right)\right] \underline{C}\left(r^{\prime}\right) \\
\times\left[\underline{I}\left(r^{\prime}\right)+\underline{Q}\left(r^{\prime}\right) \underline{S}\left(r^{\prime}\right)\right]
\end{aligned}
$$

The coupling matrix $\underline{C}$ now contains only the dipole interaction. The first-order treatment then consists of replacing $\underline{S}\left(r^{\prime}\right)$ in the integrand by $\underline{S}\left(r_{1}\right)$ and taking the limit $r \rightarrow \infty$.

Because of the strong central interaction it is preferable from a practical point of view to perform the coupledchannels integration in the region $r<r_{1}$ using the basis $\left\{\left|r \operatorname{lmSM} M_{S} I M_{I}\right\rangle\right\}$, where the electron spins are coupled to $S$ and the proton spins to $I$. This basis has as an additional advantage that it is now possible to refrain from the Shizgal approximation by including the electronic spatial degrees of freedom through the use of HeitlerLondon wave functions. Furthermore, we are now able to include both the intra-atomic as well as the interatomic hyperfine interaction. At $r=r_{1}$ we finally transform back to the asymptotic (hyperfine) basis and apply Eq. (33) only to the open channels.

\section{RESULTS AND DISCUSSION}

\section{A. Coupled channels}

In view of magnetic traps for hydrogen atoms with relevant temperatures eventually in the $10-100 \mu \mathrm{K}$ range, we present in Fig. 1 the $T=0$ rates

$$
G_{\alpha \beta \rightarrow \alpha^{\prime} \beta^{\prime}}=4 \pi^{3} \hbar^{2} \mu p_{\alpha^{\prime} \beta^{\prime}}\left|T_{\left\{\alpha^{\prime} \beta^{\prime}\right\} l^{\prime} m^{\prime},\{\alpha \beta\} 00}\left(p_{\alpha^{\prime} \beta^{\prime}}, 0\right)\right|^{2},
$$

for all 45 exothermic spin transitions in $\mathbf{H}+\mathbf{H}$ collisions as a function of magnetic field. As implied by the theory of Sec. III, the effect of the condensate is left out of consideration. The difference with previous results ${ }^{4}$ is due to a smaller integration step size and an improved $\mathbf{H}-\mathbf{H}$ singlet potential, describing more accurately the experimental $\mathrm{H}_{2}$ rovibrational energy data. Although these corrections are rather small, they accumulate in the case of exchange transitions and in some cases lead to significant changes in the rate constants of up to $50 \%$. The changes of the dipolar rates relative to Ref. 4 are only at the $10^{-3}$ level.

In view of a recent neutral trap experiment ${ }^{3}$ it is of interest to calculate also the $T \neq 0$ rates for the decay of $d d$. 
In Fig. 2 we present the three most important ones for $B=0$ [(2): $d d \rightarrow a c$; (3): $d d \rightarrow a a$; (4): $d d \rightarrow a d]$ and their weighted sum, the loss rate (curve 1). In the following subsection we present simple closed expressions for the $B$ and $T$ dependence of these and other dipolar rates.

As a second application we now consider the cryogenic $\mathrm{H}$ maser. In a previous paper ${ }^{5}$ we presented ratelike constants $\widetilde{G}$ which govern the maser spin-exchange frequency shift, calculated both by coupled channels and by simplified treatments. Rate constants $G$ of the type considered in the present paper are also of interest for the operation of the $\mathrm{H}$ maser. For instance, $b d \rightarrow a a$, $c c \rightarrow b d$, and $c c \rightarrow a a$ processes are of fundamental importance in the description of the cryogenic $\mathbf{H}$ maser operating at about $0.5 \mathrm{~K}$. With the usual spin-exchange tuning of the cavity only the part of the $\mathrm{H}+\mathrm{H}$ collisional frequency shift proportional to the difference $n_{a}-n_{c}$ of $|a\rangle$ and $|c\rangle$ atom densities can be eliminated. In order to devise strategies for eliminating fluctuations in the hyperfine-induced collisional part, ${ }^{5}$ which depends in different way on $n_{a}, n_{b}, n_{c}$, and $n_{d}$, it is necessary to calculate the latter from the rate constants $G_{\alpha \beta \rightarrow \alpha^{\prime} \beta}$. In calculating these, we neglect the very weak magnetic field within the maser. The results are presented in Fig. 3. We conclude that $G_{c c \rightarrow a a}$ and $G_{b d \rightarrow a a}$ start from nonvan- ishing values at $T=0$. However, for the transition $c c \rightarrow b d$, which is impossible at zero temperature and magnetic field, the rate increases as $T^{1 / 2}$ and becomes comparable to $G_{b d \rightarrow a a}$ at the operating temperature of the cryogenic $\mathrm{H}$ maser.

\section{B. Simple approaches}

For a description of rates in terms of simple approaches it is useful to distinguish three field regimes.

At low magnetic fields $(B \leqslant 0.1 \mathrm{~T})$ we make use of the fact that differences in internal energies are sufficiently small to apply the degenerate-internal-states approximation. Both the $T \neq 0$ rates of Figs. 2 and 3, and the $T=0$ rates in Fig. 1 (spin-exchange and dipolar) can then be reproduced by simple expressions based on this approximation. Equations (12) and (13) show how the relevant $T$-matrix elements can then be expressed in terms of pure singlet and triplet quantities: we expand the initial and final spin states in singlet and triplet parts and calculate the separate transition amplitudes using more easily calculable triplet and/or singlet phase shifts and radial wave functions.

For exchange transitions we thus get the simple formulas

$$
\begin{aligned}
& S_{\left\{\alpha^{\prime} \beta^{\prime} \mid l^{\prime} m^{\prime},\{\alpha \beta \mid l m\right.}=\left.\delta_{l^{\prime} l} \delta_{m^{\prime} m}\left(\frac{p_{\alpha^{\prime} \beta^{\prime}}}{p}\right)^{l^{\prime}+1 / 2} \mid \frac{p_{\alpha \beta}}{p}\right]^{l+1 / 2} \\
& \times \frac{1}{2}\left\langle\left\{\alpha^{\prime} \beta^{\prime}\right\}\left|\mathcal{P}^{(1)}-\mathcal{P}^{(0)}\right|\{\alpha \beta\}\right\rangle\left\{\exp \left[2 i \delta_{l}^{(1)}(p)\right]-\exp \left[2 i \delta_{l}^{(0)}(p)\right]\right\}, \\
& G_{\alpha \beta \rightarrow \alpha^{\prime} \beta^{\prime}}=\left\langle\frac{\pi \hbar^{2}}{\mu p_{\alpha \beta}} \sum_{l}(2 l+1)\left|\frac{p_{\alpha^{\prime} \beta^{\prime} p_{\alpha \beta}}}{p^{2}}\right|^{2 l+1} \sin ^{2}\left[\delta_{l}^{(1)}(p)-\delta_{l}^{(0)}(p)\right]\right\rangle_{\mathrm{th}}\left|\left\langle\left\{\alpha^{\prime} \beta^{\prime}\right\}\left|\left(\mathcal{P}^{(1)}-\mathcal{P}^{(0)}\right)\right|\{\alpha \beta\}\right\rangle\right|^{2},
\end{aligned}
$$

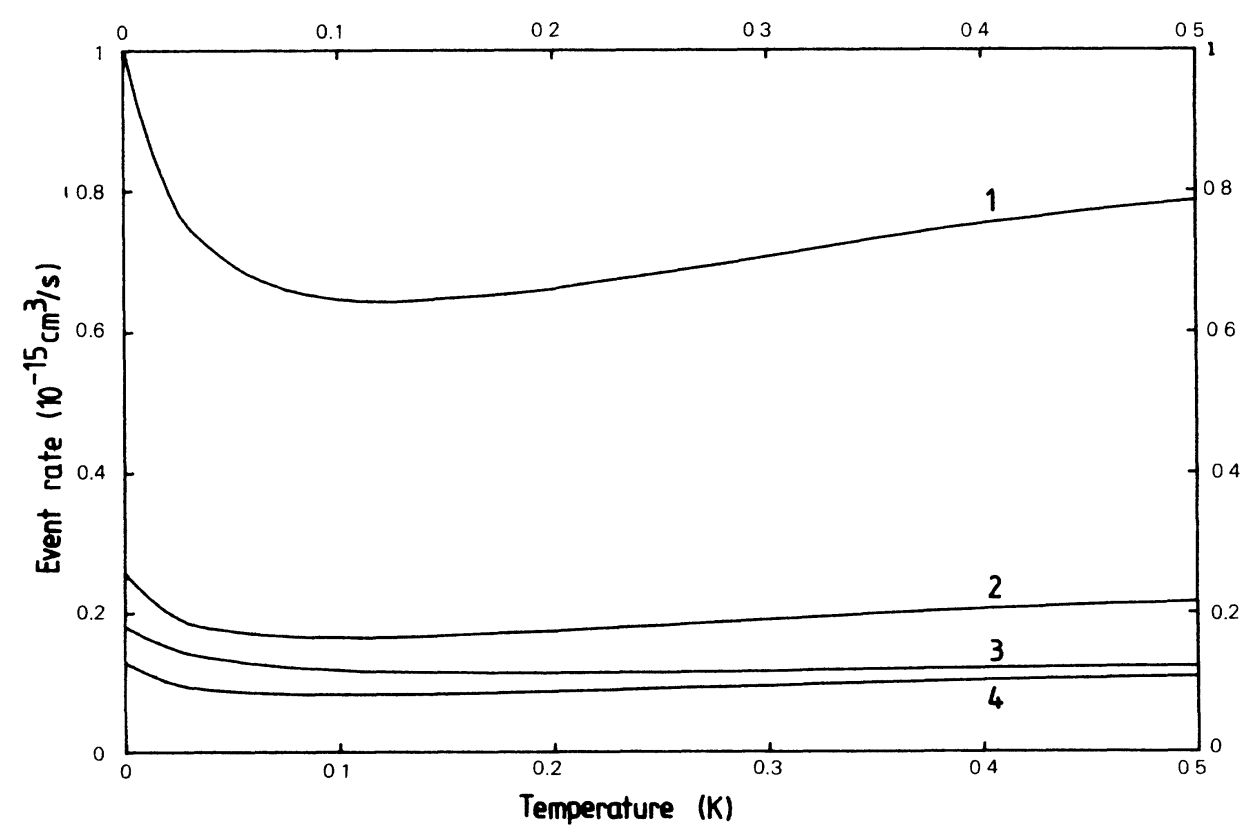

FIG. 2. Temperature dependence of three largest event rates for dipolar decay of $d d$ at $B=0$ [curve 2: $d d \rightarrow a c, 3: d d \rightarrow a a, 4:$ $d d \rightarrow a d$ ]. Curve 1: weighted sum (loss rate). Values for $B \neq 0$ follow from Eqs. (42) and (43). 


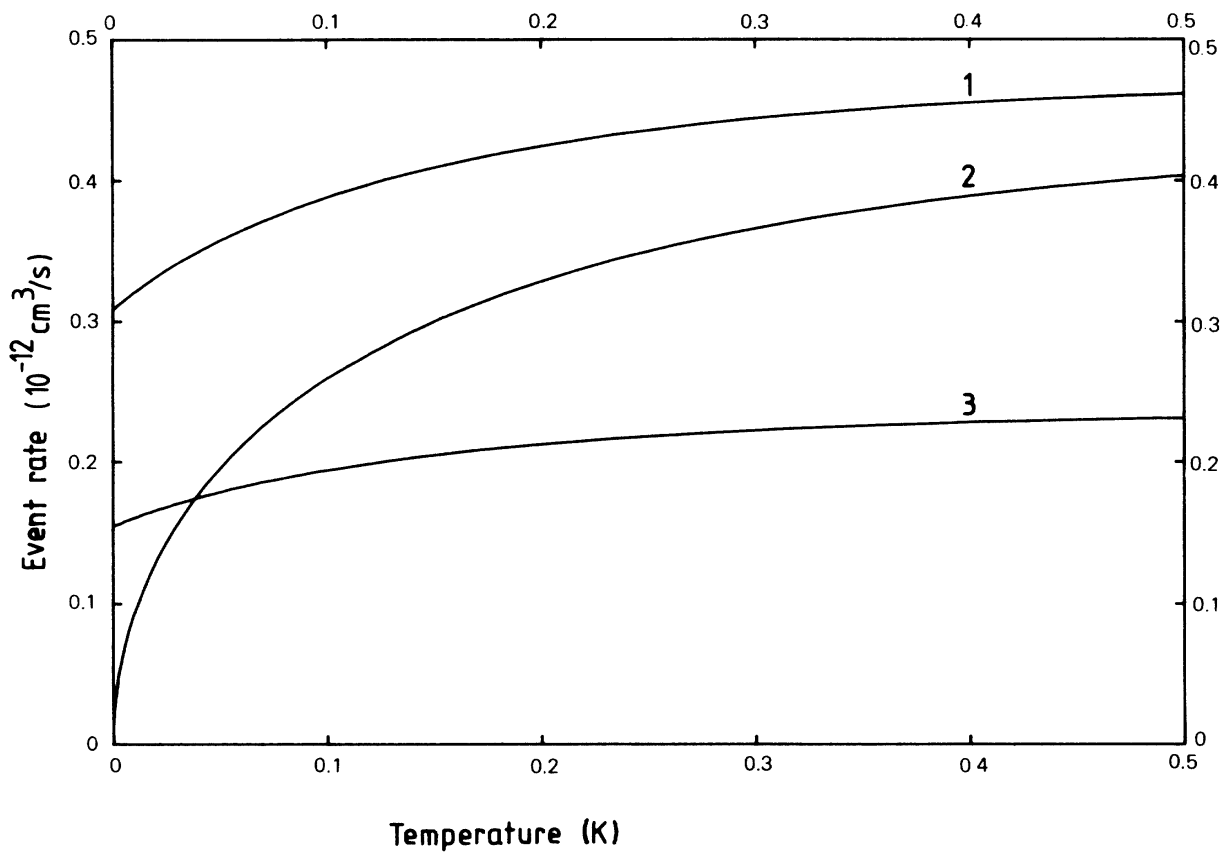

FIG. 3. Temperature dependence of three exchange event rates, important for operation of cryogenic $\mathrm{H}$ maser [curve $1: b d \rightarrow a a$, 2: $b d \rightarrow c c, 3: c c \rightarrow a a$ ]. Coupled-channels curves coincide with result based on closed formula (36).

in which some suitable expression for $p$ in terms of $p_{\alpha \beta}$ and/or $p_{\alpha^{\prime} \beta}$ can be chosen. In the limit $p \rightarrow 0$ the phaseshifts approach their effective-range limiting values:

$$
\sin \delta_{l}^{(S)}(p) \simeq \delta_{l}^{(S)}(p) \rightarrow-\delta_{l o} p a^{(S)} / \hbar
$$

with the scattering lengths denoted by $a^{(S)}$. If this choice is made for $p=p_{\alpha \beta}$ at low $T$ the result is

$$
\begin{aligned}
G_{\alpha \beta \rightarrow \alpha^{\prime} \beta^{\prime}}^{\mathrm{ex}}(T=0)= & \pi \frac{p_{\alpha^{\prime} \beta^{\prime}}}{\mu}\left(a^{(1)}-a^{(0)}\right)^{2} \\
& \times\left|\left\langle\left\{\alpha^{\prime} \beta^{\prime}\right\}\left|\left(\mathcal{P}^{(1)}-\mathcal{P}^{(0)}\right)\right|\{\alpha \beta\}\right\rangle\right|^{2},
\end{aligned}
$$

a result already given in Ref. 7 . With the values ${ }^{7}$

$$
a^{(1)}=1.34 a_{0}, \quad a^{(0)}=0.32 a_{0}
$$

for atomic hydrogen inserted, we find agreement with the rigorous $T=0$ rates in Fig. 1 up to $B=0.1 \mathrm{~T}$ to within $10 \%$. The $T \neq 0$ rates in Fig. 3 agree with the simple equation (36) with $p=p_{\alpha \beta}$ to within $5 \%$.

We stress again that the approximation proposed here allows other $p$ values to be chosen. In particular $p^{2}=p_{\alpha \beta}^{2}+2 \mu\left(\varepsilon_{\alpha}+\varepsilon_{\beta}-\varepsilon_{\alpha^{\prime}}-\varepsilon_{\beta^{\prime}}\right) / 2$ turns out to lead to highly accurate results. For $B \lesssim 0.1 \mathrm{~T}$ the results are indistinguishable from the exact ones. Furthermore, even for magnetic field strengths as high as $1 \mathrm{~T}$, the deviations are below the $10 \%$ level. We also note that the magnetic field dependence for all exchange rates is governed by factors like $p_{\alpha^{\prime} \beta^{\prime}}^{2 l+1}$ and by a simple spin matrix element.

Turning to the dipolar transitions we start from Eq. (13) and substitute $V^{d}=\Sigma_{\mu}(-1)^{\mu} V_{2 \mu}^{d} \Sigma_{2,-\mu}$ [cf. Eq. (23)]. We find

$$
T_{\left\{\alpha^{\prime} \beta^{\prime} \mid l^{\prime} m^{\prime},\{\alpha \beta\} l m\right.}=(-1)^{m^{\prime}-m} \sum_{S^{\prime}, S}\left\langle\psi_{S^{\prime} l^{\prime} m^{\prime}}^{(-)}\left(p_{\alpha^{\prime} \beta^{\prime}}\right)\left|V_{2, m^{\prime}-m}^{d}\right| \psi_{S l m}^{(+)}\left(p_{\alpha \beta}\right)\right\rangle\left\langle\left\{\alpha^{\prime} \beta^{\prime}\right\}\left|\mathcal{P}^{\left(S^{\prime}\right)} \Sigma_{2, m-m^{\prime}} \mathcal{P}^{(S)}\right|\{\alpha \beta\}\right\rangle,
$$

allowing evaluation in terms of (mixed) singlet/triplet $1 / r^{3}$ radial integrals. Apparently, the dependence on magnetic field is again located in $p_{\alpha^{\prime} \beta^{\prime}}$ and a simple spin matrix element. For the sake of brevity we give here only the spin matrix elements for the subset of dipolar transitions considered in Fig. 2:

$$
\begin{aligned}
& \left\langle\{a c\}\left|\mathcal{P}^{(1)} \Sigma^{e e e} \mathcal{P}^{(1)}\right| d d\right\rangle=2 \sqrt{3} \sin (2 \theta), \\
& \left\langle a a\left|\mathcal{P}^{(1)} \Sigma^{e e} \mathcal{P}^{(1)}\right| d d\right\rangle=2 \sqrt{6} \cos ^{2} \theta, \\
& \left\langle\{a d\}\left|\mathcal{P}^{(1)} \Sigma^{e e} \mathcal{P}^{(1)}\right| d d\right\rangle=2 \sqrt{3} \cos \theta .
\end{aligned}
$$

Comparing with the rigorous results in Fig. 1, we find numerically that the deviations are below the $4 \%$ level for $B<0.1 \mathrm{~T}$ and, as expected, increase with increasing field strength. As an illustration of Eq. (40) consider the transition $a c \rightarrow a b$ [curve 15 in Fig. 1(a)]. In this case we obtain

$$
\left\langle\{a b\}\left|\Sigma_{2,-1}^{e e}\right|\{a c\}\right\rangle=\cos (3 \theta) / \sqrt{2}
$$

and thus $G_{a c \rightarrow a b} \sim \cos ^{2}(3 \theta)$. We therefore expect $G_{a c \rightarrow a b}$ 
to have a sharp minimum at $\theta=\pi / 6$, i.e., $B \simeq 0.0292 \mathrm{~T}$. The agreement with our rigorous calculations is excellent.

Without exception the dipolar transitions are far dominated by the single term in Eq. (40) with $S^{\prime}=S=1$. This introduces a further simplification: all transition ampli- tudes contain a common orbital factor which is a "universal" function of $p_{\alpha^{\prime} \beta^{\prime}}$ and $p_{\alpha \beta}$, determined by triplet scattering. A similar conclusion therefore applies to the rates. Writing the energy $\varepsilon_{\alpha}+\varepsilon_{\beta}-\varepsilon_{\alpha^{\prime}}-\varepsilon_{\beta^{\prime}}$ released in a dipolar transition as $\Delta$, we find that for low temperatures $\left(3 k_{B} T \lesssim \Delta\right)$ the corresponding rate is described by

$$
\begin{aligned}
G_{\alpha \beta \rightarrow \alpha^{\prime} \beta^{\prime}}(B, T)= & \left(0.2130 \times 10^{-16} \mathrm{~cm}^{3} \mathrm{~s}^{-1}\right)(\Delta / a)^{1 / 2} \\
& \times\left[1-1.440 \frac{k_{B} T}{\Delta}+1.797\left[\frac{k_{B} T}{\Delta}\right]^{2}\right]\left|\left\langle\left\{\alpha^{\prime} \beta^{\prime}\right\}\left|\mathcal{P}^{(1)} \Sigma_{2, m-m^{\prime}} \mathcal{P}^{(1)}\right|\{\alpha \beta\}\right\rangle\right|^{2}
\end{aligned}
$$

to within $2 \%$. For higher temperatures $\left(0.2 \lesssim \Delta / k_{B} T \lesssim 3\right)$ we have

$$
\begin{aligned}
G_{\alpha \beta \rightarrow \alpha^{\prime} \beta^{\prime}}^{\mathrm{dip}}(B, T)= & \left(0.5741 \times 10^{-17} \mathrm{~cm}^{2} \mathrm{~s}^{-1}\right)\left(k_{B} T / a\right)^{1 / 2} \\
& \times\left[1+1.401 \frac{\Delta}{k_{B} T}-0.06181\left[\frac{\Delta}{k_{B} T}\right]^{2}\right]\left|\left\langle\left\{\alpha^{\prime} \beta^{\prime}\right\}\left|\mathcal{P}^{(1)} \Sigma_{2, m-m^{\prime}} \mathcal{P}^{(1)}\right|\{\alpha \beta\}\right\rangle\right|^{2},
\end{aligned}
$$

also to within $2 \%$. It should be pointed out that Eqs. (42) and (43) are also valid for dipolar transitions at stronger fields, provided the energy released in such a transition is not too high. In particular, the high-field behavior of the $b b \rightarrow a b$ and $a b \rightarrow a a$ dipolar transition rates, important quantities in $b$-state atom experiments, is described by Eqs. (42) and (43) with the spin matrix element equal to $-2 \sqrt{3} \mu_{p} / \mu_{e}(1+16.68 / B)$. Equation (43) is a generalization of a closed formula presented previously ${ }^{14}$ to a greater set of transitions.

In the intermediate field regime, $0.1 \mathrm{~T} \lesssim B \lesssim 1 \mathrm{~T}$, it is no longer possible to neglect the energy differences between the various hyperfine states because corrections to the $T$-matrix element to first ${ }^{5}$ and higher order in the internal level splittings become important. It appears that at these intermediate fields the solution of the full coupled-channels equation is required to obtain reliable values of the relaxation constants.

In the strong-field regime $(B \gtrsim 1 \mathrm{~T})$ a simple approach is again possible. This is already evident by a simple inspection of Fig. 1(a). Let us first pay attention to the exchange transitions. These clearly fall in three different categories: they arise essentially from cross terms of exchange and the $n$th power of the hyperfine interaction, where $n=0,1$, or 2 . This expresses itself in three different slopes $\sim B^{-2 n}$. To understand this in more detail, we consider the coupling among the channels $c c, a a,\{a c\}$, and $\{b d\}$. Ordering rows and columns correspondingly, the coupling matrix is to lowest nonvanishing order in $\theta \simeq a /\left[B\left(\mu_{e}+\mu_{p}\right)\right] \equiv \varepsilon$ :

$$
\left[\begin{array}{cccc}
V_{1} & \varepsilon^{2}\left(V_{1}-V_{0}\right) & \frac{1}{2} \sqrt{2} \varepsilon\left(V_{1}-V_{0}\right) & -\frac{1}{2} \sqrt{2} \varepsilon\left(V_{1}-V_{0}\right) \\
\varepsilon^{2}\left(V_{1}-V_{0}\right) & V_{1} & -\frac{1}{2} \sqrt{2} \varepsilon\left(V_{1}-V_{0}\right) & \frac{1}{2} \sqrt{2} \varepsilon\left(V_{1}-V_{0}\right) \\
\frac{1}{2} \sqrt{2} \varepsilon\left(V_{1}-V_{0}\right) & -\frac{1}{2} \sqrt{2} \varepsilon\left(V_{1}-V_{0}\right) & \frac{1}{2}\left(V_{1}+V_{0}\right) & \frac{1}{2}\left(V_{1}-V_{0}\right) \\
-\frac{1}{2} \sqrt{2} \varepsilon\left(V_{1}-V_{0}\right) & \frac{1}{2} \sqrt{2} \varepsilon\left(V_{1}-V_{0}\right) & \frac{1}{2}\left(V_{1}-V_{0}\right) & \frac{1}{2}\left(V_{1}+V_{0}\right)
\end{array}\right]
$$

Clearly, for strong fields the exchange coupling is in almost all cases damped by (a power of) the hyperfine mixing parameter $\varepsilon$ and can thus by treated in first order. The only exception concerns $\{b d\}$ and $\{a c\}$, which are strongly coupled mutually in a $B$ independent way. This agrees with Fig. 1(a). The $2 \times 2$ submatrix pertaining to these channels decouples if one transforms to $[\{b d\}+\{a c\}] / \sqrt{2}$ and $[\{b d\}-\{a c\}] / \sqrt{2}$ basis states. The diagonal potentials for these states are pure triplet and singlet potentials, respectively. In a sense the degenerate-internal-states approximation is again applicable here for strong fields since the difference between $b d$ and $a c$ internal energies is small. Equations (35) and (36) therefore apply. For $T \rightarrow 0$ we then have in particular

$$
G_{b d \rightarrow a c}^{\mathrm{ex}}=\pi \frac{p_{a c}}{\mu}\left(a^{(1)}-a^{(0)}\right)^{2} \cos ^{2}(2 \theta) .
$$

Equation (45) describes the $b d \rightarrow a c$ curve in Fig. 1(a) for all $B$ values to within $4 \%$.

Disregarding $O\left(\varepsilon^{3}\right)$ terms it follows also from the matrix (44) that $a a$ and $c c$ are coupled only to the difference channel $[\{b d\}-\{a c\}] / \sqrt{2}$. This explains the coincidence at higher $B$ of the 1 and 6 and of the 3 and 5 curves. In analogy to $\mathrm{Eq}$. (40) we have for these transitions

$$
\begin{aligned}
T_{\{b d\} l^{\prime} m^{\prime}, c c l m} & =-T_{\{a c\} l^{\prime} m^{\prime}, c c l m} \\
& =\delta_{l^{\prime} l} \delta_{m^{\prime} m} \frac{1}{2} \sqrt{2} \varepsilon\left\langle\psi_{0 l m}^{(-)}\left|\left(V_{1}-V_{0}\right)\right| \psi_{1 l m}^{(+)}\right\rangle \\
T_{a a l^{\prime} m^{\prime},\{b d\} l m} & =-T_{a a l^{\prime} m^{\prime},\{a c\} l m} \\
& =-\delta_{l^{\prime} l} \delta_{m^{\prime} m} \frac{1}{2} \sqrt{2} \varepsilon\left\langle\psi_{1 l m}^{(-)}\left|\left(V_{1}-V_{0}\right)\right| \psi_{0 l m}^{(+)}\right\rangle .
\end{aligned}
$$

Here we used the $S M_{S} I M_{I}$ structure of the $\alpha \beta$ states for 
strong $B$.

For weaker fields (but still so strong that the above first-order treatment applies), the singlet and triplet wave functions $\psi$ can both be inserted for zero energy, leading to four $T$-matrix elements equal in absolute magnitude. This is in agreement with Fig. 1(a): The curves 3,5 and 1,6 coincide. At somewhat stronger fields the curves separate again due to the finite final kinetic energy. From the $\varepsilon^{2}$ dependence of the $c c$-aa coupling it is finally clear that the corresponding rate falls off as $B^{-4}$.

At strong fields the dipolar transitions show a similar classification. The 11 strongest ones do not need a proton spin flip but only a single or double electron spin flip. As a consequence the transitions are almost entirely due to $V^{d, e e}$. The spin matrix element is more or less independent of $B$ (for strong fields). The highest category $(7,8,12)$ consists of electron double spin-flip transitions. The middle category $(9,13,16,17)$ of electron single spin-flip processes with $S=1$ in both initial and final states. The lowest category $(10,11,14,15)$ comprises electron single spin-flip processes where the initial or final states have $S=1$ with only $50 \%$ probability. This reduces $G$ by a factor of 2. The spin matrix element for the highest category differs by a factor $\sqrt{2}$ from that for the middle category. This is to be combined with a factor $\sqrt{2}$ from the phase-space factor $p_{\alpha^{\prime} \beta^{\prime}}$ in Eq. (34). The proportionality of the $G$ values is clearly seen in Fig. 1(a). The initial gradual increase with $B$ is due to the same factor $p_{\alpha^{\prime} \beta^{\prime}} \sim \sqrt{B}$. The explanation for the bending down of the curves at the highest $B$ values is similar as for that of curves 1 and 6 considered before: the disparity in initial and final kinetic energies. It is clear that the highest category starts to bend down at smaller $B$ : the double spin-flip energy is a factor of 2 larger.

The remaining dipolar rates all show a decreasing slope due to $\varepsilon$ and $\varepsilon^{2}$ dependences of the electron-electron spin matrix element: The dominant initial and final proton spin configurations are orthogonal. Hyperfine induced admixtures in one and two atoms, respectively, are required to achieve equal proton spin wave functions. The first category contains the $b b \rightarrow a b$ and $a b \rightarrow a a$ relaxations as special cases, and also $d d \rightarrow a c$, which is one of the most important dipolar transitions for the decay of the static magnetic trap. At the fields considered the electron-electron contribution dominates. At the higher fields electron-proton relaxation starts to take over. Depending on the relative sign of the electron-electron and electron-proton matrix elements, $G$ curves start to bend down or up relative to their above-mentioned dominant slope.

\section{CONCLUSIONS}

Given the effective Hamiltonian the coupled-channels method is a rigorous method for calculating $\mathrm{H}+\mathrm{H}$ spinexchange and dipolar rates. In the foregoing we have described this method and some of its results: the $B$ dependence of all $T=0$ rates and the $T$ dependence of some $B=0$ rates. The latter have been selected on the basis of their importance for the cryogenic $\mathrm{H}$ maser and for the $\mathbf{H} \uparrow$ magnetic trap. Closed-form formulas for the rates, useful for the analysis of both types of experiments, have been presented for the field and temperature dependence in the weak-field regime $(B \lesssim 0.1 \mathrm{~T})$, based on the degenerate-internal-states approximation. A simple interpretation has been given also for the strong-field behavior $(B \gtrsim 1 \mathrm{~T})$ of the rates.

\section{ACKNOWLEDGMENT}

This work is part of a research program of the Stichting voor Fundamenteel Onderzoek der Materie (FOM), which is financially supported by the Nederlandse Organisatie voor Zuiver Wetenschappelijk Onderzoek (ZWO), The Netherlands.
${ }^{1}$ B. R. Johnson, J. S. Denker, N. Bigelow, L. P. Lévy, J. H. Freedom, and D. M. Lee, Phys. Rev. Lett. 52, 1508 (1984).

${ }^{2}$ H. F. Hess, G. P. Kochanski, J. M. Doyle, T. J. Greytak, and D. Kleppner, Phys. Rev. A 34, 1602 (1986); M. D. Hürlimann, W. N. Hardy, A. J. Berlinsky, and R. W. Cline, ibid. 34, 1605 (1986); R. L. Walsworth, Jr., I. F. Silvera, H. P. Godfried, C. C. Agosta, R. F. C. Vessot, and E. M. Mattison, ibid. 34, 2550 (1986).

${ }^{3}$ H. F. Hess, G. P. Kochanski, J. M. Doyle, N. Masuhara, D. Kleppner, and T. J. Greytak, Phys. Rev. Lett. 59, 672 (1987).

${ }^{4}$ A. Lagendijk, I. F. Silvera, and B. J. Verhaar, Phys. Rev. A 33, 626 (1986).

${ }^{5}$ B. J. Verhaar, J. M. V. A. Koelman, H. T. C. Stoof, O. J. Luiten, and S. B. Crampton, Phys. Rev. A 35, 3825 (1987).

${ }^{6}$ R. M. C. Ahn, J. P. H. W. v. d. Eijnde, and B. J. Verhaar, Phys. Rev. B 27, 5424 (1983).

${ }^{7}$ J. M. V. A. Koelman, H. T. C. Stoof, B. J. Verhaar, and J. T.
M. Walraven, Phys. Rev. Lett. 59, 676 (1987).

${ }^{8}$ J. R. Taylor, Scattering Theory (Wiley, New York, 1972); W. Glöckle, The Quantum Mechanical Few-Body Problem (Springer-Verlag, Berlin, 1983).

${ }^{9}$ A. Messiah, Quantum Mechanics (North-Holland, Amsterdam, 1961).

${ }^{10}$ A. M. Lane and R. G. Thomas, Rev. Mod. Phys. 30, 257 (1958).

${ }^{11}$ J. Raynal, in Computing as a Language of Physics, edited by A. Salam (IAEA, Vienna, 1972), p. 292.

${ }^{12}$ W. J. G. Thijssen, B. J. Verhaar and A. M. Schulte, Phys. Rev. C 23, 1984 (1981).

${ }^{13}$ C. E. Pearson, Handbook of Applied Mathematics (Van Nostrand-Reinhold, New York, 1974).

${ }^{14}$ J. P. H. W. van den Eijnde, Ph. D. thesis, Eindhoven University of Technology, The Netherlands, 1984. 